OPEN ACCESS

Edited by:

Sigal Uziel-Karl,

Achva Academic College, Israel

Reviewed by:

Xavier Moonen,

University of Amsterdam, Netherlands

Anna Matamala,

Universitat Autònoma de Barcelona,

Spain

*Correspondence:

Julia Fuchs

fuchsj@uni-mainz.de

Specialty section:

This article was submitted to

Language Sciences,

a section of the journal

Frontiers in Communication

Received: 20 April 2021

Accepted: 23 August 2021

Published: 06 September 2021

Citation:

Borghardt L, Deilen S, Fuchs J,

Gros A-K, Hansen-Schirra S, Nagels A,

Schiffl $L$ and Sommer J (2021)

Neuroscientific Research on the

Processing of Easy Language.

Front. Commun. 6:698044.

doi: $10.3389 /$ fcomm.2021.698044

\section{Neuroscientific Research on the Processing of Easy Language}

\author{
Liv Borghardt, Silvana Deilen, Julia Fuchs *, Anne-Kathrin Gros, Silvia Hansen-Schirra, \\ Arne Nagels, Laura Schiffl and Johanna Sommer
}

Gutenberg Council for Young Researchers (GYR), Easy Language Research Group, Johannes Gutenberg University Mainz, Mainz, Germany

In an increasingly networked world, people who cannot participate in written communication are particularly at risk of social exclusion. Like other concepts of barrier-free communication, Easy Language ("Leichte Sprache") attempts to enhance perceptibility and comprehensibility of texts for people with reading impairments by means of a rule-based reduction of complexity on the text side. The underlying assumption of simplified languages is that the reduction of linguistic complexity correspondingly reduces cognitive processing costs. However, this interplay between linguistic complexity and cognitive processing costs still needs to be investigated by empirical research as up to date there are only a few studies investigating the perception and reception of Easy Language, mostly using offline methods (e.g., questionnaires or retrospective interviews). In contrast to offline methods, which are only capable of assessing comprehension products, online methods allow researchers to track what a participant is focusing their attention on at any given time and to thereby develop a detailed representation of the time-course of cognitive language processing. In our paper, we aim to point out how different online methods (eye-tracking, EEG and fMRI) can be used for investigating the empirical validity of the postulated rules for Easy Language by evaluating cognitive processing efficiency. Besides discussing the applicability of those neuroscientific online methods in Easy Language research, we discuss the importance of collecting personal and neuropsychological data to gain detailed profiles of the participants and therefore not only contribute to the explanation of variance but furthermore to determine the role of neuropsychological skills on reading proficiency. For each online method we elaborate basic principles, discuss some of the main findings in cognitive sciences and demonstrate the greatest advantages but also restrictions of the method and challenges related to the data collection process with impaired participants. Furthermore, we outline current challenges in Easy Language research and summarize remaining research gaps. On a final note, we emphasize that it is both the establishment of online methods and the data triangulation in Easy Language research that enable researchers to gain a profound insight into the cognitive processing of simplified languages.

Keywords: Easy Language, simplified languages, written language processing, cognitive processing costs, neurolinguistic methods, communicative impairments 


\section{INTRODUCTION}

Easy Language is conceived as a variety of a language that is reduced systematically on different linguistic levels, for example on the lexical and syntactic level (cf. Maaß, 2015). On a continuum with different comprehensibility levels represented by specific language forms, Easy Language is characterized by a maximum comprehensibility level and constitutes one extreme pole of the continuum. The other extreme pole, the elaborate level, is represented by languages for special purposes. Plain Language and standard language are located between these two extreme poles, plain language having an intermediary comprehensibility level and standard language being the standard level (cf. Maaß, 2020: 51). Easy Language has initially been developed for individuals with intellectual and learning disabilities who have difficulties with understanding regular texts written in standard language. Today, however, the target groups are significantly larger, comprising also individuals with dementia, prelingual hearing impairment and aphasia as well as functional illiterates and people with German as a second language (Bredel and Maaß, 2016a: 140-172).

A major challenge Easy Language research is currently facing consists in the empirical investigation of the effectiveness of the different rules postulated by the guidelines for Easy Language, for example, with regard to German Easy Language, by the early practical guidelines (Inclusion Europe, 2009; ${ }^{1}$ Netzwerk Leichte Sprache, 2009; BITV 2.0, 2011 ${ }^{3}$ ) and by the scientifically founded guidelines (Maaß, 2015; Bredel and Maaß, 2016a; Bredel and Maaß, 2016b; cf. Maaß, 2020: 69-87). It is thus necessary to investigate the comprehension of Easy Language. In linguistics and its neighbouring disciplines, the different methods used for the investigation of comprehension can be subdivided into two groups: online methods on the one hand and offline methods on the other. Online methods measure "the processes that come into play in comprehension itself" (Gillioz and Zufferey, 2020: 17); in this context, Christmann (2002) uses the term processes of comprehension. Examples of online methods are eye-tracking, electroencephalography (EEG) or functional magnetic resonance imaging (fMRI). On the other hand, offline methods "affect the final interpretations resulting from the comprehension process" (Gillioz and Zufferey, 2020: 17); for designating the object of offline methods, Christmann (2002) uses the term products of comprehension. Examples of offline methods are questionnaires or recall tasks.

The empirical investigation of the effectiveness of different rules on Easy Language has so far mainly been restricted to the use of offline methods (e.g., Lange, 2019). In contrast, only very few studies have addressed the comprehension of Easy Language using online methods (but: Pappert and Bock, 2019; Fuchs et al. submitted manuscript $)^{4}$. Even though " $[\mathrm{t}]$ here are no good or bad measures in experimental linguistics" (Gillioz and Zufferey, 2020:

${ }^{1}$ https://www.lag-abt-niedersachsen.de/uploads/migrate/Download/Infofralle.pdf. ${ }^{2}$ https://www.leichte-sprache.org/wp-content/uploads/2017/11/Regeln_Leichte_ Sprache.pdf.

${ }^{3}$ http://www.gesetze-im-internet.de/bitv_2_0/BJNR184300011.html.

${ }^{4}$ Fuchs, J., Schaeffer, M., Hansen-Schirra, S. Do adults with and without Intellectual Disabilities Benefit from German Easy Language? Eye-Tracking and Recall Studies on the Processing of Causal and Conditional Relations. Submitted Manuscript
19), processes of comprehension can only be investigated via online methods - and it is in this field that the most significant current research gaps in Easy Language research are located. As an important basis for addressing these desiderata, the present article provides the relevant background information about online methods allowing to investigate processes of comprehension in the context of Easy Language and its target groups. The article is structured as follows: Metadata and Test Battery section shows which metadata and neuropsychological information about the participants should be collected; the following sections focus on the online methods eye-tracking (Eye-Tracking section), EEG section and fMRI section and their application in the context of Easy Language research. In the final section, the strengths and problems of online methods in the context of Easy Language are summarized.

\section{METADATA AND TEST BATTERY}

\section{Metadata}

Since the target groups for Easy Language are heterogeneous within and across groups it is important to collect metadata concerning the participants. Similarly to other psycholinguistic experiments, general data such as age, sex, native language etc. are collected. This information is important because factors such as native language or educational level can impact the level of reading experience which in turn influences reading skills. However, additional data come into play when involving participants with special communication needs. Information on the kind and degree of disability is important in order to differentiate the target groups. Differentiating the target groups is necessary to analyze cognitive factors that impact reading ability. Furthermore, the degree of disability is one of the main factors influencing an individual's level of reading ability.

Comparable to other psycholinguistic experiments and tests, data collection has to be carried out on the basis of informed consent. In the case of persons not capable of giving consent (e.g., children, some of the people with cognitive disability or dementia), their legal representative will be asked to sign the form. In case they are able to give consent, information on the experiment has to be presented in Easy or Plain Language to ensure comprehensibility.

\section{Neuropsychological Testing}

As the target group of German Easy Language with its several subgroups is expected to be very heterogeneous concerning not only the recipients' abilities to process written language but also their cognitive performance, neuropsychological skills should be evaluated precisely when investigating language processing with regard to Easy Language.

In the course of variance explanation, the participants' neuropsychological performance is to be regarded as of the same importance as their metadata (as discussed above). Therefore, a neuropsychological test battery should be implemented in each experimental design using online methods to examine cognitive processing. A minimum of the following subtests and their constructs are recommended to be 
taken into consideration. As the discussed tests are suitable for German native speakers they may need to be adapted to the examined language. Equivalent or similar tests that measure corresponding abilities should be used and are commonly available in neuropsychological assessment batteries for the relevant language.

\section{Psychomotor Ability and Mental Flexibility}

Psychomotor ability, speed of processing and mental flexibility can be assessed by the Trail Making Test (TMT-A and TMT-B). The TMT-A tests for psychomotor ability and speed of processing. The widely used diagnostic instrument originates in the Army Individual Test Battery (1944), where it was used for diagnosing attention disorders. The TMT is a paper and pencil test, where participants are asked to connect numbers (1-25) in ascending order by drawing a connective line without lifting the pen off the paper. The second part of the TMT (TMT-B) additionally takes mental flexibility into account as participants are asked to not only connect numbers but also letters in alternating order (numbers 1-13, letters A-L). For both parts, participants' performance is evaluated by the time taken to complete the task. In order to pass the test, participants are required to take no longer than $3 \mathrm{~min}$ for each part. In consequence, participants with a slow cognitive processing speed will likely fail the TMT-A and the TMT-B.

\section{Working Memory}

As the working memory capacity is an important factor in processing written language (e.g., Ober et al., 2019), it should be taken into investigation for each individual. One possible way to do so is through having participants repeat a series of numbers (Zahlenspanne). This is done in numerous test batteries (e.g., WAIS-R by Wechsler, 1981). In the test "Zahlenspanne" the reproduction of a number series by the participants is queried forwards (2-9 digits) in the first and backwards (2-8 digits) in a second part and allows conclusions about the participants' auditory memory span. The auditory processing abilities are particularly relevant for a successful acquisition of written language (Wise et al., 2010; Pezzino et al., 2019) and should be considered as highly relevant when assessing a neuropsychological profile of the target group. Considering that people with intellectual disability are reported to show a specific deficit in the phonological memory span (Schuchardt et al., 2011), working memory might take on an important role in the reading abilities and should be carefully surveyed. Psycholinguistic studies have found that the working memory span of unimpaired adults has an average size of six to eight items (digits, words or other units) depending on the phonological complexity of these (Miller, 1956; Hulme et al., 1995).

\section{Word Fluency}

Word fluency is considered a factor of verbal intelligence. It can be measured through the Regensburger Wortflüssigkeitstest (RWT - "Regensburger Word Fluency Test" by Aschenbrenner et al., 2000). Participants are given a category and are asked to orally list as many words as possible in $1 \mathrm{~min}$. Three categories are considered in the RWT: Semantic (e.g., animals), lexical/ phonemic (e.g., words beginning with the letter "p") and mixed semantic (e.g., alternating "fruits" and "sports"). By analyzing participants' ability to generate words, conclusions can be drawn about the size and organization of the mental lexicon as well as their cognitive strategies for successful and fast word retrieval (Whiteside et al., 2016).

\section{Verbal Intelligence}

For verbal intelligence the Mehrfachwahl-WortschatzIntelligenztest (MWT-B - "Multiple-Choice Vocabulary Intelligence Test" by Lehrl, 2005) can be used. Participants are asked to select one existing word within a word selection of nonwords. In each line four, non-words are presented with one real word. The number of recognized words, out of the whole of 37 lines, then gives feedback on vocabulary size and verbal intelligence. It should be taken into account though, that because of the MWT-B being a written assignment, participants with low literacy skills might find special challenges solving the test.

A further analysis and correlation of the described neuropsychological testing as well as the metadata with processing data described in the further sections could possibly be used in the attempt of defining determinants for the reading and processing abilities of individuals with communicative impairments. This kind of data triangulation furthermore contributes to explaining the statistical variation among the target groups. Besides the factors described above, Hansen-Schirra and Maaß (2020: 29) suggest testing the target groups' abilities concerning hand-eye coordination, visual processing speed, visual-spatial abilities, general level of intelligence and reading speed. While those abilities can also be relevant, researchers working with Easy Language target groups should consider the duration of the experiments themselves and of the pretesting in order to avoid overstraining the participants mentally and/or physically. It is important to consider that certain challenges can occur when dealing with Easy Language target groups and to carefully select the tests suitable for your specific research question. The whole test battery should not be too extensive. In addition, some of the tests will possibly not be applicable due to the limitations in reading abilities and comprehension abilities or sensory limitations of several participants.

\section{EYE-TRACKING}

Eye-tracking is one of the most widely used techniques to investigate cognitive processes in reading (for an overview see e.g., Rayner, 1992; Rayner, 1998).

Most of the currently available eye-trackers are infrared videobased tracking systems mounted beneath or integrated into a display screen. The camera emits infrared light which is directed at the eyes. After entering the retina, a large proportion of the light is reflected and captured by image sensors. Subsequently, an eye gaze analysis software is used to calculate eye movements, gaze direction and fixation points. As it is assumed that the time the participant spends fixating on a word equals the time the word 
is being processed (a phenomenon known as "eye-mind assumption," cf. Just and Carpenter, 1980), fixations are used as an indicator of participants' focus of attention. Therefore, the fixation position indicates, at least to a certain extent, which part of the sentence is currently processed. As a longer fixation duration is usually associated with a deeper or more effortful cognitive processing, the time the eyes remain on and return to this position is also indicative of the difficulty in extracting word information. Researchers are consequently able to identify passages, words or even letters that increase cognitive processing costs which in turn allows them to draw inferences about the underlying psychological processes during reading. To ensure that the comprehension process was successful, those drawn inferences from the eye-tracking experiment can then be checked against results from further comprehension tests, such as text-picture-matching or follow-up questions.

Since several studies have shown that eye movements are directly influenced by textual variables - for example, increased linguistic complexity leads to increased fixation duration and decreased saccade length - eye-tracking is especially suited to investigate the empirical validity of the postulated rules for Easy Language. In recent years, more and more researchers are taking advantage of this when evaluating the effectiveness of Easy Language rules.

Three eye-tracking studies are currently being conducted by the research group "Simply complex - Easy Language." Two of them investigate processing costs at the interface of morphology and lexis: Schiffl (2020) focuses on the effects of a target word's frequency, length and number of occurrences in the text, while Deilen (2020) infers differences in the cognitive processing of compounds that are segmented with a hyphen, segmented with a mediopoint or not segmented at all. As both studies involve the target groups of Easy Language - adults with cognitive impairment and students with prelingual hearing impairment - they face similar challenges conducting and carrying out the eye-tracking data acquisition (see Deilen and Schiffl, 2020). Eyetracking was also used in the study conducted by Fuchs et al. (submitted manuscript) on the processing of causal and conditional relations by adults with and without intellectual disabilities.

Other researchers like Wellmann (2021) and Gutermuth (2020) also have implemented eye-tracking research with one or more of the Easy Language target groups to evaluate reading and comprehension processes. Similarly to Deilen (2020), Wellmann (2021) sets out to answer the question of how different segmentation signs (namely hyphen and mediopoint) affect the processing of compounds in Easy Language. Her study was conducted with representatives of the target group "learners of German as a second language." Gutermuth (2020) investigates the reception and processing difficulties of authentic texts with varying complexity levels (including Plain Language) for people with cognitive impairment, people with migration background and seniors. For her study she combined eye-tracking with tests of comprehensibility and recall.

One of the main advantages of using eye-tracking technology to investigate cognitive processing of Easy Language is that many of the currently available systems are mobile. Since there is no need for participants to leave their familiar surroundings, researchers can conduct their studies at different locations, thus reaching many of the heterogenous target groups of Easy Language. However, researchers investigating cognitive processing within the Easy Language target groups should be aware of several challenges when conducting their experiments. Many times, participants with cognitive impairment (due to mental disabilities, dementia, aphasia or other circumstances) will not be capable of the same requirements as unimpaired participants. To avoid mentally and/or cognitively overstraining the participants, conductors should carefully monitor the experiment's duration. Furthermore, participants with impairment cannot be expected to show the same ability in terms of understanding and acting in accordance to experimental instructions. Instructions should therefore be held rather simple than complicated and tasks should not involve complex steps or combinations (e.g., "if sentence $\mathrm{x}$ is true, press button 1; if not, press button 2"). In terms of self-control, participants with cognitive impairment might face difficulties, making it harder to sit quietly in front of a computer screen while restricting head and body movement. Even if instructions are well understood, participants with impairment might need more guidance and reminders in comparison to unimpaired adults. Also, participants without impairment, especially university students, will usually have an easier time dealing with the unnatural situation of (reading) experiments. Lastly, on a more practical level, physical impairments concerning the eyes (e.g., squint, nystagmus, thick glasses) seem to occur more often within the group of intellectually impaired people, making the calibration of the eye-tracking-system more difficult (cf. Splunder et al., 2006; Csakvari and Gyori, 2015).

\section{EEG}

The Electroencephalogram (EEG) has been a useful technique in neurophysiological, psycholinguistic and clinical linguistic research, as well as neuropsychological diagnostics. Since the 1920s, researchers measure electric activity on the human scalp by means of active electrodes. The positive or negative signals derive from summed postsynaptic polar shifts from pyramidal cells perpendicular to the cortical surface (cf. Luck, 2014). When a participant perceives auditory or visual stimuli, the neurotransmissions move to the scalp surface. Depending on the study, a differing number of active or passive electrodes pick up the voltage fluctuations. The potentials connected to a specific event are called event related potentials (ERPs) and appear as soon as a participant perceives a stimulus. Therefore, EEG has a high temporal resolution and is highly efficient in research dealing with the time course of stimuli processing.

To measure ERPs, active or passive electrodes are connected to an amplifier from which the signal is sent to a computer (Luck, 2014: 21). Because of the high sensitivity to muscle and eye movements, eye blinks, heart beat and power signal artefacts, eye electrodes are usually placed next to the eyes and experiments 
take place in a soundproof cabin. The raw data has to be preprocessed before it can be analyzed. Processing steps include filtering of the frequency bandwidth, decomposition into independent components (ICA) so that muscle and eyeblink artefacts are erased from the signal, and baseline correction. Then, the data can be segmented into the relevant time windows associated with a critical stimulus. The analysis methods for ERPs differ from time-frequency-analysis. The extraction of ERPs happens by averaging trial amplitude values of same experimental conditions for each participant in a first step and grand-averaging amplitudes of conditions across all participants in a second step. The pairwise-comparisons between the conditions across all participants can then be undertaken by a cluster-based permutation test. Here, the amplitude values of the two conditions are compared and significant differences between conditions for each time point in each electrode position can be detected.

Whereas ERPs are sensitive to the time domain, timefrequency-analysis adds a further dimension to the observations. Here, the EEG signal is decomposed in five frequency bands of interest that range from $<4 \mathrm{~Hz}$ (delta band) up to $80 \mathrm{~Hz}$ (gamma band, $30-80 \mathrm{~Hz}$ ) and analyzed according to an event (event related oscillation, c.f. Tamm, 2005: 8-12).

The extracted ERPs can give insights into a wide range of brain responses towards stimuli. Participants' responses towards uncanonical sentence structures or morpho-syntactic violations (classically reported as a positive voltage shift $600 \mathrm{~ms}$ after the stimulus (Osterhout and Holcomb, 1992: 791) can be observed as well as participants' expectancy towards an auditory or visual stimulus. A word's frequency, familiarity or phonological complexity modulates the activation level and therefore is more or less expected in a sentence. These effects have been reported as enhanced negativity after $400 \mathrm{~ms}$ post onset an unexpected stimulus (cf. Kutas and Hillyard, 1980a: 103, Kutas and Hillyard, 1980b: 203-205, Kutas and Federmeier, 2011: 622-644 for a review). Over the past decades, a huge psycholinguistic research field has expanded observing components such as the P300, N400 and P600 creating a complex mass of theories on the interaction of context with the incremental integration of words into a sentence on all linguistic levels. By means of the components, the relationship between cognitive processing efficiency and a participant's comprehension of an Easy Language product can be examined (e.g., Van Petten and Luka, 2012 for a review on prediction). For instance, the use of words with higher frequency could be reflected in a decrease of the N400 component. This would imply that the brain required less processing costs in order to process the word on a semantic level. Another effect could be diminished brain activity due to syntactic complexity reduction when readers process Easy Language material. If there was no processing cost decrease when an adult control group had read Easy Language sentences, the syntactic structure of the stimulus should be revised. Increasingly, also time-frequency-analysis has been employed in language processing studies (Prystauka and Lewis, 2019: 5-31 for a review). For instance, the processing of semantic anomalies have been found in increased theta oscillations (3-7 Hz) (e.g., Bastiaansen and Hagoort, 2015: 2,100-2,103, and gamma band oscillation (>30 Hz) (e.g., Hald et al., 2006: 95-98) as well as decrease in beta power $(16-19 \mathrm{~Hz})$ (Wang et al., 2012: 2904-2906). Whereas a wide range of questions on interlingual comparisons, clinical research questions and research on second language learners has been observed (e.g., Hahne, 2001; Midgley et al., 2009; Barkley et al., 2015; Almor et al., 2017), to our knowledge no research has been undertaken exploring the processing of reduced or simplified language such as Plain English or German Easy Language using the EEG methodology so far. In Korean, Kang et al. (2017) investigated the influence of intelligence level (high or low) on the processing of visuo-spatial and language tasks with two difficulty levels. Measuring alpha, beta and theta band coherence, the results showed that individuals with different cognitive preconditions processed the stimuli of the two tasks differently (Kang et al., 2017: 51ff.). Even though the study is not a linguistic one on simplified Korean, it reveals that the results obtained in the time-frequency-domain potentially reflect specific linguistic aspects in a text that lead to increased processing costs for specific target groups.

Because of the well-established research on language processing through EEG, the method can serve as a useful tool for modeling complexity stages in processing simplified language. Theoretically, there is no restriction to be included in EEG experiments and all types of stimuli can be presented. Furthermore, the method can be combined e.g., with eyetracking or fMRI. The extraction of ERPs and the analysis of the time-frequency domain make it possible to draw conclusions on very specific language phenomena and therefore built models on the complexity of language processing for simplified languages as well. When testing members of the target group, insights into interacting processes such as attention, memory and language processes can be observed with EEG. Potentially, predictors for the usefulness of simplified language can be detected.

However, several aspects need to be considered when planning an EEG study with target groups of Easy Language. Participants with cognitive impairments are more likely to have a lower attention span as compared to unimpaired groups and thus might have difficulties following the instructions. Also, movement artefacts are more likely for this group. Reading assessments, neuropsychological tests (see Chapter 2) and additional behavioral measures are crucial for those participants to control for effects associated with cognitive impairments. It is also important to consider possible methodological constraints. When presenting a whole text to participants in reading studies, eye movements during reading cause strong artefacts that distort the ERP recording. Therefore, text stimuli must be presented one word at a time, either in rapid serial visual presentation (RSVP) or self-paced reading mode. However, RSVP cannot be considered a natural reading situation as participants have no control over the input. Additionally, in order to get a better resolution of the ERP components, words are usually presented at slow rates (500-800 ms per word), while in natural reading, readers generally read five words per second. The prolonged duration of the word presentation may cause interpretive processes in the reader that would be reflected in 
the ERPs (Camblin et al., 2007: 176-178). Self-paced reading is considered more natural than RSVP and has been employed in ERP studies (e.g. Ditman et al., 2007). Nevertheless, it may cause eye movement artefacts and may increase the probability of interpretive processes. Furthermore, it can be a problematic task for subjects with cognitive disabilities. To bypass this problem, auditory presentation is used in many studies. Some findings show that components are sensitive to input modality, for instance the N400. Holcomb and Neville (1990) found that the N400 occurred earlier and lasted longer with auditory than with written stimuli presentation. Additionally, the scalp distribution is different for the two modalities (Holcomb and Neville, 1990: 296-301). Therefore, it has to be ensured that the participants have sufficient reading skills so that the stimuli can be visually presented.

In general, it has to be considered that parts of the target group might face difficulties taking part in an EEG study. Even though EEG is a non-invasive method, the necessity of sitting in a soundproof booth without being allowed to move makes the situation ethically critical. For some target groups the demands might be bearable, e.g., for prelingually hearing impaired readers (cf. Malaia et al., 2020). However, for people with a high degree of disability, EEG is not feasible, since the length of experiments might be too effortful and the placement of the electrodes on the scalp is physically challenging. Also, the repetition of stimulus material demands a high amount of attention and concentration. These aspects have to be considered when planning studies on language forms for and with a specific target group.

\section{FMRI}

Functional magnetic resonance imaging (fMRI) is a neuroimaging technique that uses MRI scanners to investigate changes in brain function. MRI is based on the use of a very strong magnetic field, which energetically excites hydrogen atoms in the body. The energy emitted later can be measured and localized. This allows anatomic structures to be depicted clearly. It does not involve radiation and therefore an MRI-survey is harmless and can be repeated frequently (cf. Huettel et al., 2009: 3-21, 35). In fMRI-experiments, a three-dimensional anatomical image of the head is constructed first using the (structural) MRI. The brain activity detected in the fMRI is transferred to this image as intensity-coded colour areas at a later stage (cf. Huettel et al., 2009: 91-97, 369-372). This determination of brain areas with increased activation is the aim of fMRI. It is a hemodynamic method, which means that changes of the concentration of oxygen in the blood are measured. This measurement is based on the so-called neurovascular coupling, which describes the relationship between the activity of nerve cells and subsequent changes in cerebral blood flow. Since the brain does not provide storage capacities for energy, any energy consumption must be compensated directly. Active neural regions must therefore be provided with energy carriers via the blood flow (cf. Huettel et al., 2009: 165-166, 176-178).

The main energy carrier in all metabolic cycles is oxygen, which is transported through the blood via hemoglobin.
Increased activity in a region causes increased blood flow into that region. This changes the distribution of oxygenated and deoxygenated hemoglobin; there is even an excess of oxygen. Deoxygenated hemoglobin is paramagnetic and leads to greater inhomogeneity in the magnetic field (cf. Huettel et al., 2009: 201-211). This is the basis of the Blood Oxygenation LevelDependent response (short: BOLD response), which associates the respective brain activity with the task performed by the participant of an experiment.

Hence, local changes in the hemodynamic function are measured while a participant performs an experimental task. In order to depict such brain functions, images are constructed that distinguish the activity levels from areas of the brain. These images are based on the functional contrast described above. It is important to note that in all cases, differences between two contrasts with statistically significant differences and no absolute activations are described (cf. Huettel et al., 2009: 11).

FMRI is a comparatively new method and has only been applied since the early 1990s. Nevertheless, it has become well established in linguistic investigations. Especially in the field of semantic and syntactic investigations, several studies have already been conducted. In these studies, the focus was not only on localizing production and processing (e.g., Dapretto and Bookheimer, 1999; Friederici et al., 2000; Friederici et al., 2003; Heim et al., 2003), but also on the differences in the demands on the brain in the processing of simple vs. complex constructions (e.g., Just et al., 1996; Röder et al., 2002; Müller et al., 2003). Yet, there are still many open questions concerning brain functions. Even analyses with the same object of study do not always yield comparable results. It should also be noted that many functions are based on distributed networks (e.g., the DualStream-Model, cf. Hickok and Poeppel, 2007) and that a single brain region is involved in more than one function (e.g., Broca's area, cf. Fiebach et al., 2005). Accordingly, fMRI studies must always be interpreted critically in the sense that activated brain areas could also be attributed to other functions that may be triggered by the stimulus material, e.g., an active button press task involves motor regions or the working memory is activated during sentence processing (the so-called reverse inference, $\mathrm{cf}$. also Huettel et al., 2009: 490-491).

As mentioned, several studies on complexity in language have been published, but no fMRI studies in the field of Easy Language research have been conducted yet. However, since the rules mentioned at the beginning (see Introduction) build on assumptions about cognitive demands and working memory performance, such investigations are necessary, especially on the syntactic level. Although some well-known studies are transferable (e.g., studies of word order such as Röder et al., 2002), further studies, e.g., on maximum sentence complexity and above all based on the Easy Language rules, can advance research. Similar to the components in the EEG (see EEG section), stimuli formulated in Easy Language may cause a decrease in the BOLD response (compared to more complex conditions), leading to the corresponding conclusion that these structures imply a lower demand in processing. However, it is important to note that attention, especially in a control group, can decrease after some 
time. In order to keep it high, comprehension questions can be included, which can, for instance, be answered by a button press. In this way, data on the comprehensibility of the presented stimuli is obtained simultaneously.

It should be noted, however, that studies with at least some of the target groups are not feasible. The ethical acceptability is not clear, since an fMRI investigation requires some effort for the participant. Not only the length of the investigation, during which the participant is positioned in the scanner the whole time and is not allowed to move, but also the safety risks resulting from the strong magnetic field are problematic. Even if MRI does not pose any danger to the participants when used correctly, the strong and rapidly changing magnetic fields during scanning result in several safety challenges (cf. Huettel et al., 2009: 44). Hence, before each scan procedure there is an instruction concerning the risks, which must be understood and approved. It must therefore be ensured that this is really understood. Furthermore, the length of an experiment also affects concentration and attention. The shown tasks or stimuli must be presented repeatedly (30-50 reps/ condition), which means a high demand on the participant. The type of presentation of the stimuli must also be adapted respectively (see also EEG section on EEG).

Nonetheless, fMRI studies focusing on Easy Language are important for the further development and validation of the existing rules. Experiments with unimpaired participants can also help to investigate current assumptions about the processing of transformed texts and to develop a neurobiologically-feasible model for the processing of Easy Language.

\section{CONCLUSION}

A major gap Easy Language research is currently facing consists in the empirical investigation of the effectiveness of the existing rules using online methods. The research community has to enhance its understanding of the comprehension processes triggered by Easy Language in order to be able to decide whether and in what way the existing rules have to be revised or not. In psycho- and neurolinguistics, comprehension processes are investigated via online methods like eye-tracking, EEG and fMRI. At present, first studies using online methods in the context of Easy Language research are being conducted with unimpaired participants and with different target groups. But to the best of our knowledge, these studies are restricted to the investigation of German Easy Language. The present article aimed at giving a first

\section{REFERENCES}

Almor, A., Nair, V. A., Boiteau, T. W., and Vendemia, J. M. C. (2017). The N400 in Processing Repeated Name and Pronoun Anaphors in Sentences and Discourse. Brain Lang. 173, 52-66. doi:10.1016/ j.bandl.2017.06.003

Army Individual Test Battery (1944). Manual of Directions and Scoring. Washington DC: War Department, Adjutant General's Office.

Aschenbrenner, S., Tucha, O., and Lange, K. (2000). Regensburger Wortflüssigkeits-Test (RWT). Göttingen, Bern. Toronto, Seattle: Hogrefe, Verlag für Psychologie. overview over the application of the online methods eye-tracking, EEG and fMRI in the context of Easy Language research and stressed the importance of collecting metadata and neuropsychological information about participants. The strengths of online methods lie in their potential to give insights into the real-time processing of language, in contrast to offline methods such as questionnaires, which are only capable of informing about comprehension products. Mobile systems, mostly eye-trackers, enable researchers to collect data in the field. However, researchers are also facing several challenges when using online methods: due to the special neuropsychological dysfunctions of most target groups, the length of the experiments has to be restricted to a reasonable extent. This can be problematic if several conditions requiring numerous stimuli are of interest. Moreover, researchers have to make sure that the instructions are well understood in particular by the target groups. Any data loss due to the specific dispositions of the target groups, e.g., due to impairments concerning the eyes causing difficulties with the calibration of an eye-tracker, must already be considered in the planning phase of an experiment. It is advisable to recruit more participants from target groups of Easy Language than in usual studies with unimpaired populations.

Researchers are called on to make their experiences with online methods in the context of Easy Language research available to the community so that this kind of research can be established and continuously improved. The present article makes a first step into this direction and is intended to provide (initial) guidance for designing such studies.

\section{AUTHOR CONTRIBUTIONS}

SH-S developed the conception and design of the manuscript. LB, $\mathrm{SD}, \mathrm{JF}, \mathrm{A}-\mathrm{KG}$, LS, and JS wrote the manuscript and edited the final version. AN supervised the project and the paper writing. All authors contributed to the paper and the final version of the manuscript has been approved for submission by all authors: they are accountable for the whole work.

\section{FUNDING}

This research was funded by the Gutenberg Council for Young Researchers (GYR).

Barkley, C., Kluender, R., and Kutas, M. (2015). Referential Processing in the Human Brain: An Event-Related Potential (ERP) Study. Brain Res. 1629, 143-159. doi:10.1016/j.brainres.2015.09.017

Bastiaansen, M., and Hagoort, P. (2015). Frequency-based Segregation of Syntactic and Semantic Unification during Online Sentence Level Language Comprehension. J. Cogn. Neurosci. 27 (11), 2095-2107. doi:10.1162/jocn_a_00829

BITV 2.0 (2011). Barrierefreie-Informationstechnik-Verordnung, Anlage 2, Teil 2. Web Available at: http://www.gesetze-im-internet.de/bitv_2_0/BJNR184300011. html. (Accessed April 12, 2021).

Bock, B. M. (2019). "Leichte Sprache" - Kein Regelwerk. Sprachwissenschaftliche Ergebnisse und Praxisempfehlungen aus dem LeiSA-Projekt (KommunikationPartizipation - Inklusion 5). Berlin: Frank \& Timme. 
Bredel, U., and Maaß, C. (2016a). Leichte Sprache. Theoretische Grundlagen. Orientierung für die Praxis (Sprache im Blick). Berlin: Dudenverlag.

Bredel, U., and Maaß, C. (2016b). Ratgeber Leichte Sprache. Die wichtigsten Regeln und Empfehlungen für die Praxis (Sprache im Blick). Berlin: Dudenverlag.

Camblin, C. C., Ledoux, K., Boudewyn, M., Gordon, P. C., and Swaab, T. Y. (2007). Processing New and Repeated Names: Effects of Coreference on Repetition Priming with Speech and Fast RSVP. Brain Res. 1146, 172-184. doi:10.1016/ j.brainres.2006.07.033

Christmann, U. (2002). Methoden der Verstehens- und Verständlichkeitserhebung. Z. Literaturwiss Linguistik 32 (4), 76-97. doi:10.1007/BF03379326

Csákvári, J., and Gyori, M. (2015). Applicability of Standard Eye-Tracking Technique in People with Intellectual Disability: Methodological Conclusions from a Series of Studies. Stud. Health Technol. Inform. 217, 63-70. Print.

Dapretto, M., and Bookheimer, S. Y. (1999). Form and Content. Neuron 24, 427-432. doi:10.1016/s0896-6273(00)80855-7

Deilen, S. (2020). "Easy Language Research : Text and User Perspectives," in Easy Language Research: Text and User Perspectives. Editors S. Hansen-Schirra and C. Maaß (Berlin: Frank \& Timme), 241-256. doi:10.26530/20.500.12657/42088

Deilen, S., and Schiffl, L. (2020). "Easy Language Research : Text and User Perspectives," in Easy Language Research: Text and User Perspectives. Editors S. Hansen-Schirra and C. Maaß (Berlin: Frank \& Timme), 273-281. doi:10.26530/20.500.12657/42088

Ditman, T., Holcomb, P. J., and Kuperberg, G. R. (2007). An Investigation of Concurrent ERP and Self-Paced reading Methodologies. Psychophysiology 44, 927-935. doi:10.1111/j.1469-8986.2007.00593.x

Fiebach, C. J., Schlesewsky, M., Lohmann, G., von Cramon, D. Y., and Friederici, A. D. (2005). Revisiting the Role of Broca's Area in Sentence Processing: Syntactic Integration versus Syntactic Working Memory. Hum. Brain Mapp. 24, 79-91. doi:10.1002/hbm.20070

Friederici, A. D., Meyer, M., and Cramon, D. Y. v. (2000). Auditory Language Comprehension: An Event-Related fMRI Study on the Processing of Syntactic and Lexical Information. Brain Lang. 75, 465-477. doi:10.1006/brln.2000.2438

Friederici, A. D., Rüschemeyer, S.-A., Fiebach, C. J., and Hahne, A. (2003). The Role of Left Inferior Frontal and superior Temporal Cortex in Sentence Comprehension: Localizing Syntactic and Semantic Processes. Cereb. Cortex 13, 170-177. doi:10.1093/cercor/13.2.170

Gillioz, C., and Zufferey, S. (2020). Introduction to Experimental Linguistics (Cognitive Science Series). Hoboken, NJ: John Wiley \& Sons.

Gutermuth, S. (2020). Eine zielgruppenorientierte Rezeptionsstudie zu Leichter und Einfacher Sprache. Berlin: Frank \& Timme.One for All?

Hahne, A. (2001). What's Different in Second-Language Processing? Evidence from Event-Related Brain Potentials. J. Psycholinguistic Res. 30, 251-266. doi:10.1023/A:1010490917575

Hald, L. A., Bastiaansen, M. C. M., and Hagoort, P. (2006). EEG Theta and Gamma Responses to Semantic Violations in Online Sentence Processing. Brain Lang. 96 (1), 90-105. doi:10.1016/j.bandl.2005.06.007

Hansen-Schirra, S., and Maaß, C. (2020). "Easy Language Research : Text and User Perspectives," in Easy Language Research: Text and User Perspectives. Editors S. Hansen-Schirra and C. Maaß (Berlin: Frank \& Timme), 17-38. doi:10.26530/ 20.500.12657/42088

Heim, S., Opitz, B., Müller, K., and Friederici, A. D. (2003). Phonological Processing during Language Production: fMRI Evidence for a Shared Production-Comprehension Network. Cogn. Brain Res. 16, 285-296. doi:10.1016/S0926-6410(02)00284-7

Hickok, G., and Poeppel, D. (2007). The Cortical Organization of Speech Processing. Nat. Rev. Neurosci. 8 (5), 393-402. doi:10.1038/nrn2113

Holcomb, P. J., and Neville, H. J. (1990). Auditory and Visual Semantic Priming in Lexical Decision: A Comparison Using Event-Related Brain Potentials. Lang. Cogn. Process. 5, 281-312. doi:10.1080/01690969008407065

Huettel, S. A., Song, A. W., and McCarthy, G. (2009). Functional Magnetic Resonance Imaging. Sunderland, Massachusetts: Sinauer Associates.

Hulme, C., Roodenrys, S., Brown, G., and Mercer, R. (1995). The Role of LongTerm Memory Mechanisms in Memory Span. Br. J. Psychol. 86 (4), 527-536. doi:10.1111/j.2044-8295.1995.tb02570.x

Inclusion Europe (2009). Informationen für alle. Europäische Regeln, wie man Informationen leicht lesbar und verständlich macht. Available at:
https://www.lag-abt-niedersachsen.de/uploads/migrate/Download/Infofralle.pdf (Accessed April 10, 2021).

Just, M. A., and Carpenter, P. A. (1980). A Theory of Reading: From Eye Fixations to Comprehension. Psychol. Rev. 87 (4), 329-354. doi:10.1037/0033295x.87.4.329

Just, M. A., Carpenter, P. A., Keller, T. A., Eddy, W. F., and Thulborn, K. R. (1996). Brain Activation Modulated by Sentence Comprehension. Science 274, 114-116. doi:10.1126/science.274.5284.114

Kang, J.-S., Ojha, A., Lee, G., and Lee, M. (2017). Difference in Brain Activation Patterns of Individuals with High and Low Intelligence in Linguistic and Visuo-Spatial Tasks: An EEG Study. Intelligence 61, 47-55. doi:10.1016/j.intell.2017.01.002

Kutas, M., and Federmeier, K. D. (2011). Thirty Years and Counting: Finding Meaning in the N400 Component of the Event-Related Brain Potential (ERP). Annu. Rev. Psychol. 62, 621-647. doi:10.1146/annurev.psych.093008.131123

Kutas, M., and Hillyard, S. A. (1980a). Event-related Brain Potentials to Semantically Inappropriate and Surprisingly Large Words. Biol. Psychol. 11, 99-116. doi:10.1016/0301-0511(80)90046-0

Kutas, M., and Hillyard, S. (1980b). Reading Senseless Sentences: Brain Potentials Reflect Semantic Incongruity. Science 207, 4427203-4427205. doi:10.1126/ science. 7350657

Lange, D. (2019). Der Genitiv in der "Leichten Sprache" - das Für und Wider aus theoretischer und empirischer Sicht. Z. für Angew. Linguistik 70 (1), 37-72. doi:10.1515/zfal-2019-2001

Lehrl, S. (2005). Mehrfachwahl-Wortschatz-Intelligenztest (MWT-B). 5. Unveränderte Auflage. Balingen: Spitta Verlag.

Luck, S. (2014). An Introduction to the Event-Related Potential Technique. Cambridge, MA, United States: MIT Press.

Maaß, C. (2020). Balancing Comprehensibility and Acceptability (Easy - Plain Accessible 3). Berlin: Frank \& Timme. doi:10.26530/20.500.12657/42089Easy Language - Plain Language - Easy Language Plus : Balancing Comprehensibility and Acceptability

Maaß, C. (2015). Leichte Sprache. Das Regelbuch (Barrierefreie Kommunikation 1). Münster: LIT.

Malaia, E. A., Krebs, J., Roehm, D., and Wilbur, R. B. (2020). Age of Acquisition Effects Differ across Linguistic Domains in Sign Language: EEG Evidence. Brain Lang. 200, 104708. doi:10.1016/j.bandl.2019.104708

Midgley, K. J., Holcomb, P. J., and Grainger, J. (2009). Language Effects in Second Language Learners and Proficient Bilinguals Investigated with Event-Related Potentials. J. Neurolinguist. 22 (3), 281-300. doi:10.1016/j.jneuroling.2008.08.001

Miller, G. A. (1956). The Magical Number Seven, Plus or Minus Two: Some Limits on Our Capacity for Processing Information. Psychol. Rev. 63 (2), 81-97. doi:10.1037/h0043158

Müller, R.-A., Kleinhans, N., and Courchesne, E. (2003). Linguistic Theory and Neuroimaging Evidence: an fMRI Study of Broca's Area in Lexical Semantics. Neuropsychologia 41, 1199-1207. doi:10.1016/S0028-3932(03)00045-9

Netzwerk Leichte Sprache (2009). Die Regeln für Leichte Sprache. Available at: https://www.leichte-sprache.org/wp-content/uploads/2017/11/Regeln_Leichte_ Sprache.pdf (Accessed April 12, 2021).

Ober, T. M., Brooks, P. J., Plass, J. L., and Homer, B. D. (2019). Distinguishing Direct and Indirect Effects of Executive Functions on Reading Comprehension in Adolescents. Reading Psychol. 40, 551-581. doi:10.1080/ 02702711.2019.1635239

Osterhout, L., and Holcomb, P. J. (1992). Event-Related Brain Potentials Elicited by Syntactic Anomaly. J. Mem. Lang. 31, 785-806. doi:10.1016/0749-596X(92)90039-Z

Pezzino, A.-S., Marec-Breton, N., and Lacroix, A. (2019). Acquisition of Reading and Intellectual Development Disorder. J. Psycholinguist Res. 48 (3), 569-600. doi:10.1007/s10936-018-9620-5

Prystauka, Y., and Lewis, A. G. (2019). The Power of Neural Oscillations to Inform Sentence Comprehension: A Linguistic Perspective. Lang. Linguist Compass 13, 9. doi:10.1111/lnc3.12347

Rayner, K. (1992). Eye Movements and Visual Cognition: Scene Perception and reading. New York: Springer-Verlag.

Rayner, K. (1998). Eye Movements in reading and Information Processing: 20 Years of Research. Psychol. Bull. 124, 372-422. doi:10.1037/0033-2909.124.3.372

Röder, B., Stock, O., Neville, H., Bien, S., and Rösler, F. (2002). Brain Activation Modulated by the Comprehension of normal and Pseudo-word Sentences of Different Processing Demands: A Functional Magnetic Resonance Imaging Study. NeuroImage 15, 1003-1014. doi:10.1006/nimg.2001.1026 
Schiffl, L. (2020). "Easy Language Research : Text and User Perspectives," in Easy Language Research: Text and User Perspectives. Editors S. Hansen-Schirra and C. Maaß (Berlin: Frank \& Timme), 227-239. doi:10.26530/20.500.12657/ 42088

Schuchardt, K., Maehler, C., and Hasselhorn, M. (2011). Functional Deficits in Phonological Working Memory in Children with Intellectual Disabilities. Res. Dev. disabilities 32 (5), 1934-1940. doi:10.1016/j.ridd.2011.03.022

Tamm, Sascha. (2005). Hochaufgelöste Zeit-Frequenz-Analysen Ereigniskorrelierter EEG-Oszillationen Mittels S-Transformation. dissertation. Berlin: Freie Universität. doi:10.17169/refubium-11564

Van Petten, C., and Luka, B. J. (2012). Prediction during Language Comprehension: Benefits, Costs, and ERP Components. Int. J. Psychophysiology 83, 176-190. doi:10.1016/j.ijpsycho.2011.09.015

van Splunder, J., Stilma, J. S., Bernsen, R. M. D., and Evenhuis, H. M. (2006). Prevalence of Visual Impairment in Adults with Intellectual Disabilities in the Netherlands: Cross-Sectional Study. Eye 20, 1004-1010. doi:10.1038/sj.eye.6702059

Wang, L., Jensen, O., van den Brink, D., Weder, N., Schoffelen, J.-M., Magyari, L., et al. (2012). Beta Oscillations Relate to the N400m during Language Comprehension. Hum. Brain Mapp. 33, 2898-2912. doi:10.1002/hbm.21410

Wechsler, D. (1981). Wechsler Adult Intelligence Scale-Revised. New York: Psychological Corporation.

Wellmann, K. (2021). "Medio-punkt mit Binde-Strich? Eine EyetrackingStudie," in Leichte Sprache - Empirische und multimodale Perspektiven. Editors A. Gros, S. Gutermuth, and K. Oster (Berlin: Frank \& Timme), $23-42$.
Whiteside, D. M., Kealey, T., Semla, M., Luu, H., Rice, L., Basso, M. R., et al. (2016) Verbal Fluency: Language or Executive Function Measure? Appl. Neuropsychol. Adult 23, 29-34. doi:10.1080/23279095.2015.1004574

Wise, J. C., Sevcik, R. A., Romski, M., and Morris, R. D. (2010). The Relationship between Phonological Processing Skills and Word and Nonword Identification Performance in Children with Mild Intellectual Disabilities. Res. Dev. disabilities 31 (6), 1170-1175. doi:10.1016/j.ridd.2010.08.004

Conflict of Interest: The authors declare that the research was conducted in the absence of any commercial or financial relationships that could be construed as a potential conflict of interest.

Publisher's Note: All claims expressed in this article are solely those of the authors and do not necessarily represent those of their affiliated organizations, or those of the publisher, the editors and the reviewers. Any product that may be evaluated in this article, or claim that may be made by its manufacturer, is not guaranteed or endorsed by the publisher.

Copyright (c) 2021 Borghardt, Deilen, Fuchs, Gros, Hansen-Schirra, Nagels, Schiff and Sommer. This is an open-access article distributed under the terms of the Creative Commons Attribution License (CC BY). The use, distribution or reproduction in other forums is permitted, provided the original author(s) and the copyright owner(s) are credited and that the original publication in this journal is cited, in accordance with accepted academic practice. No use, distribution or reproduction is permitted which does not comply with these terms. 